\title{
Using information technology to control STls
}

\author{
Christopher K Fairley
}

Sexual Health Unit, Melbourne School of Population Health, The University of Melbourne and Melbourne Sexual Health Centre, Alfred Health, Victoria, Melbourne, Australia

Correspondence to Christopher Fairley, Melbourne Sexual Health Centre, 580 Swanston Street, Carlton, Victoria 3053, Australia; cfairley@unimelb.edu.au

Accepted 24 February 2011

This paper is freely available online under the BMJ Journals unlocked scheme, see http://sti. bmi.com/site/about/unlocked. xhtml

\section{INTRODUCTION}

Information technology (IT) could potentially decrease the rate of sexually transmitted infections (STIs) by increasing uptake of STI testing and treatment, increasing condom use or, possibly, by reducing partner change. This article will focus on the use of practical IT interventions from a clinic or health department perspective (table 1). The use of IT outside clinics or health departments is beyond the scope of this article, but clearly many avenues are available to facilitate behaviour change, not least, of course, being the rapidly expanding phenomena of social media. ${ }^{1}$

The sexual history provides the risk assessment on which STI testing is based and is well suited to the use of computer-assisted self-interviewing (CASI). ${ }^{2}$ CASI can overcome inequalities and disadvantage through the use of audio for those who cannot read or though translation into other languages. Studies comparing CASI to clinician histories have clearly shown that CASI provides more accurate answers to social desirability questions. ${ }^{2}$ What is not established yet is if the routine use of CASI results in improved uptake of testing and treatment, which is essential if it is to be an effective intervention to reduce the rates of STI. ${ }^{2}$

The outcome of CASI on testing and treatment has been evaluated in only two studies in STI clinics. In one three-arm randomised control trial (RCT) of 2351 STI clinic attendees in the UK, HIV testing was $27 \%$ lower in those randomised to CASI compared to those randomised to see a clinician. ${ }^{3} 4$ The second study was an observational study of over 14000 individuals analysed from a clinic where CASI was operating as part of routine care. In this study, chlamydia testing was lower in the period when CASI was operating compared to the period when it was not ( $84 \%$ vs $88 \%$, respectively) but HIV testing rates were not different in these two periods. ${ }^{4}$ Importantly, in neither of these two studies were STI testing rates significantly higher in patients who underwent CASI compared to seeing a clinician. ${ }^{3} 4$

One potential advantage of CASI is that it may shorten the duration of a clinical consultation and, therefore, allow more patients to be seen (and therefore tested) with the same resources than if CASI were not in place. The effect of CASI on the time of clinical consultations has been investigated in only one study. ${ }^{4}$ This study showed that there were no significant differences in the mean duration of consultations. The study had $80 \%$ power to rule out differences of greater than 2 min in consultation time. ${ }^{4}$

Another issue that is relevant to CASI within STI services is whether they can elicit more symptoms, particularly if CASI is planned to completely replace clinical consultations. This has been assessed in two studies and both suggest that CASI is less sensitive than a clinician history for the detection of acute symptoms. ${ }^{5}$ Given the importance of symptoms in directing testing and treatment, these two studies suggest that the use of CASI in complete isolation from clinicians may have disadvantages. However, a number of centres are using CASI in combination with express consultations but these have yet to be evaluated. ${ }^{7}$

The electronic medical record (EMR) has the potential to make a substantial contribution to the quality of sexual healthcare and, therefore, improving testing and treatment of STIs. There is clear evidence that the recording of consultations in EMRs is superior to their paper counterparts but EMRs also allow other quality improvement and time savings measures such as electronic prescribing. ${ }^{8}$ In developing countries, EMRs have also been shown to be useful with one before and after analysis of remote HIV clinics showing significant reductions in waiting time and clinical consultation times. ${ }^{9}$ With EMRs there is also the potential for automated quality reports for clinicians to improve their testing and treatment performance. But, importantly, if designed well, the EMR provides the opportunity to support clinical decisions through decision support software. ${ }^{10}$ However, the EMR will only work well in sexual health medicine if it includes the right data fields to allow these alerts to work. For example, if an alert is to remind clinicians to vaccinate men who have sex with men (MSM) there must be a field in the software for the sex of the client and the sex of their partners. The combination of CASI inputting the relevant data fields into the EMR provides a particularly efficient method for incorporating the relevant data with an EMR.

Decision support software, including simple electronic prompts, has been found to be highly effective in other areas of medicine. ${ }^{10}$ In sexual health, a simple alert increased chlamydia testing by $32 \%$ in a cluster RCT in general practices. ${ }^{11}$ In another study, a simple clinician alert increased syphilis testing by $15 \%$ (from $77 \%$ to $89 \%$ ) among high risk MSM. ${ }^{12}$ The increase was also associated with a higher proportion of syphilis cases being diagnosed when they were asymptomatic suggesting that the alert prompted earlier diagnosis and, therefore, reduced the infectious period. ${ }^{12}$

IT has also been used to increase access to counselling services. In two RCTs in US emergency departments, the provision of information for pre and post HIV test counselling was equally effective when provided by a counsellor or through audio assisted CASI. ${ }^{13}$ In another RCT, self-reported condom use was significantly higher in those exposed to two 15-40 min computer-based motivational interviewing sessions than in those exposed 
Table 1 Potential uses of information technology to facilitate sexually transmitted infection (STI) control within clinics

\begin{tabular}{|c|c|}
\hline Information technology & Benefit, potential benefit or harm \\
\hline Computer-assisted self-interviewing & $\begin{array}{l}\text { Provides more accurate risk assessment (E) } \\
\text { May increase STI testing (NE) } \\
\text { - May increase efficiency (NE) } \\
\text { - Reduces symptom reporting (E) } \\
\text { - Provides automatic data fields for EMR to allow decision support software to operate }\end{array}$ \\
\hline Electronic Medical Record (EMR) & $\begin{array}{l}\text { Improves quality of recorded information }(\mathrm{E}) \\
\text { Allows programming of DSS } \\
\text { - Allows automated quality assurance monitoring }\end{array}$ \\
\hline Decision support software (DSS) & $\begin{array}{l}\text { Benefits well-studied in other areas of medicine (eg, prescribing errors) (E) } \\
\text { Increases STI screening through clinician alerts (E) }\end{array}$ \\
\hline Computer-based counselling & $\begin{array}{l}\text { Increases knowledge }(\mathrm{E}) \\
\text { Reduces self-reported STI risk }(\mathrm{E}) \\
\text { - Acceptable }(\mathrm{E})\end{array}$ \\
\hline Short text messages (SMS) to clients & $\begin{array}{l}\text { Improves appointment attendance (E) } \\
\text { - Improves STI screening (E) }\end{array}$ \\
\hline Web-based STI testing & - Attracts high risk individuals for testing (E) \\
\hline $\begin{array}{l}\text { Web-based partner notification services allow anonymous notification } \\
\text { of sexual partners }\end{array}$ & - Widely used and rarely abused (E) \\
\hline
\end{tabular}
of sexual partners

E, established with at least observational studies with a control period or control group; NE, no published data available or insufficient published information to know if the IT achieves benefit or harm.

to the control arm $(\mathrm{p}<0.05) .{ }^{14}$ These data together and the finding that a significant proportion of STI clinic attendees are comfortable with some computer-based counselling indicate that it may have considerable potential to reduce STI rates. ${ }^{15}$

However, there has been insufficient work to know how to incorporate counselling using IT into STI services. The Project RESPECT established that two 20 min interactive counselling sessions were more effective for reducing future STI acquisition than brief didactic sessions. ${ }^{16}$ However, the important IT question is whether computer-based behavioural interventions are as effective (or cost effective) as the standard established in project RESPECT. Even if computer-assisted counselling was less effective than project RESPECT, because computer-assisted counselling is very significantly cheaper it may be much more cost effective at a population level. While ideally all at risk clients should be provided with standard project RESPECT counselling, it is unlikely that many STI services have the capacity to provide this particularly in the current dire funding environment. ${ }^{17}$

IT can also be used effectively during clinic dead time when, for example, clients are waiting to be seen. An innovative US multisite study assessed the effect of health promotion videos running continuously in waiting areas and showed that these were associated with a $10 \%$ reduction in notifiable STIs. ${ }^{18}$ These resources are freely available and one could argue that clinic directors need a reason not to install these resources.

IT has the potential to completely change the way services are provided. In the US, Gaydos et al set up a website where individuals can request STI testing kits (http://www.iwantthekit. org/). ${ }^{19}$ Over a 4-year period between 2004 and 2008, 3774 kits were requested of which $32.4 \%$ were returned. Of these, 1203 participants, $64.0 \%$ were black, the median age was 23 years and the chlamydia prevalence was high at $9.1 \% .{ }^{19}$

Other websites have taken alternative approaches with IT and tried to assist general practitioners (GPs) with STI testing and treatment. One website uses individual responses to a detailed sexual history to generate recommended STI tests that are then taken to a GP. ${ }^{20}$ In another intervention, a partner notification web address was frequently access by GPs when it appeared on positive STI results. $^{21}$

Increasingly, a number of private medical services are offering clinical services through the web. For example, Dr Thom in the UK will post you a urine collection kit for chlamydia and gonorrhoea testing without a consultation for $£ 47.99$ or for $£ 30$ you can speak with a GP for up to $15 \mathrm{~min}$ (https://www. drthom.com/). The role of these services in STI control has not been evaluated but in one US study the addition of a US\$15 surcharge was associated with a $29 \%$ decline in attendances and the decline was more pronounced among higher risk individuals. ${ }^{22}$

Perhaps one of the most important uses of IT is to facilitate partner notification. In addition to providing educational material these websites allow individuals to contact their partners anonymously either through email or short text messages (SMS). The first of these websites was set up in San Francisco in the USA (http://www.inspot.org/). ${ }^{23}$ Universally they have been shown to be cheap, widely used and rarely abused. ${ }^{23}$ Their aim is not to replace other forms of partner notification, but allow partners who would not have been contacted to be informed of their possible STI risk and, therefore, reduce STI burden.

IT has a major role in improved efficiency and effectiveness of existing clinical services either through simply improving access to information such as treatment guidelines on the web or through automating tasks. The finance industry has clearly demonstrated the dramatic cost savings that can result when IT is adopted. For example, the cost of a transaction at a bank is US\$1.10 at a teller, US\$0.30 at an automatic teller machine and less than 1 cent over the internet. Sexual health services are adopting ways to increase efficiency and now give results through automated text messages or remind patients about the need for retesting. In one study at a sexual health service, MSM who received an SMS reminder were over four times more likely to be retested than those who did not. ${ }^{24}$

A critical part of many IT interventions within health services is ensuring there is adequate IT infrastructure. This infrastructure is a prerequisite for CASI, electronic health records, decision support software, computer-based counselling, automated quality reports, automated SMS to clients about appointments or the need for retesting. Given the increasing evidence that these IT features of a health service, contribute to or directly result in an increase in their efficiency and effectiveness, health departments should facilitate the necessary infrastructure over the next few years. Finally, careful and meticulous evaluation of the effectiveness of each IT intervention will greatly assist governments as they allocate scarce public funds for the greatest public good. 
Competing interests None declared.

Contributors The author of this paper was solely responsible for the content.

Provenance and peer review Commissioned; externally peer reviewed.

\section{REFERENCES}

1. Vance K, Howe W, Dellavalle RP. Social internet sites as a source of public health information. Dermatol Clin 2009:27:133-6, vi.

2. Fairley CK, Sze JK, Vodstrcil LA, et al. Computer-assisted self interviewing in sexual health clinics. Sex Transm Dis 2010;37:665-8.

3. Richens J, Copas A, Sadiq ST, et al. A Three-arm Randomised Controlled Trial Comparing Computer-Assisted Self-interview With Computer-assisted Physician Interview And Pen And Paper Face-to-face Sexual History Taking In A Clinic Setting [Abstract: 0-02]. The British Association for Sexual Health and HIV \& American STD Association (BASHH ASTDA) Meeting. New York, 2008

4. Vodstrcil LA, Hocking JS, Cummings $\mathrm{R}$, et al. Computer assisted self interviewing in a sexual health clinic as part of routine clinical care; impact on service and patient and clinician views. PLoS One 6(3):e18456. doi:10.1371/journal.pone.0018456.

5. Kurth AE, Martin DP, Golden MR, et al. A comparison between audio computer-assisted self-interviews and clinician interviews for obtaining the sexual history. Sex Transm Dis 2004;31:719-26.

6. Rogers SM, Willis G, Al-Tayyib A, et al. Audio computer assisted interviewing to measure HIV risk behaviours in a clinic population. Sex Transm Infect 2005;81:501-7.

7. Camberwell Sexual Health Centre. King's College Hospital-NHS Fundation Trust. Denmark Hill, London. http://www.kch.nhs.uk/services/specialist-medicine/ sexual-health/camberwell-sexual-health-centre (accessed 23 Sep 2009).

8. Hippisley-Cox J, Pringle M, Cater R, et al. The electronic patient record in primary care - regression or progression? A cross sectional study. BMJ 2003;326:1439-43.

9. Fraser HS, Biondich P, Moodley D, et al. Implementing electronic medical record systems in developing countries. Inform Prim Care 2005;13:83-95.

10. Garg AX, Adhikari NK, McDonald H, et al. Effects of computerized clinical decision support systems on practitioner performance and patient outcomes: a systematic review. JAMA 2005;293:1223-38.

11. Walker J, Fairley CK, Walker SM, et al. Computer reminders for Chlamydia screening in general practice: a randomized controlled trial. Sex Transm Dis 2010;37:445-50.
12. Bissessor M, Fairley CK, Leslie D, et al. Use of a computer alert and increased testing of high risk men who have sex with men for syphilis within a sexual health service. Australasian Sexual Health Conference. Sydney, Australia, 2010.

13. Calderon $\mathbf{Y}$, Leider J, Hailpern $\mathrm{S}$, et al. A randomized control trial evaluating the educational effectiveness of a rapid HIV posttest counseling video. Sex Transm Dis 2009:36:207-10.

14. Kiene SM, Barta WD. A brief individualized computer-delivered sexual risk reduction intervention increases HIV/AIDS preventive behavior. J Adolesc Health 2006; 39:404-10.

15. Weinhardt LS, Mosack KE, Swain GR. Development of a computer-based riskreduction counseling intervention: acceptability and preferences among low-income patients at an urban sexually transmitted infection clinic. AIDS Behav 2007:11:549-56

16. Kamb ML, Fishbein M, Douglas JM Jr, et al. Efficacy of risk-reduction counseling to prevent human immunodeficiency virus and sexually transmitted diseases: a randomized controlled trial. Project RESPECT Study Group. JAMA 1998:280:1161-7.

17. Golden MR, Kerndt PR. Improving clinical operations: can we and should we save our STD clinics? Sex Transm Dis 2010;37:591-2.

18. Warner L, Klausner JD, Rietmeijer CA, et al; Safe in the City Study Group. Effect of a brief video intervention on incident infection among patients attending sexually transmitted disease clinics. PLoS Med 2008;5:e135.

19. Gaydos CA, Barnes M, Aumakhan B, et al. Can e-technology through the Internet be used as a new tool to address the Chlamydia trachomatis epidemic by home sampling and vaginal swabs? Sex Transm Dis 2009;36:577-80.

20. Lee DM, Fairley CK, Sze JK, et al. Access to sexual health advice using an automated, internet-based risk assessment service. Sex Health 2009;6:63-6.

21. Tomnay JE, Pitts MK, Fairley CK. General practitioners' use of internet-based patient materials for partner notification. Sex Transm Dis 2007;34:613-16.

22. Rietmeijer CA, Alfonsi GA, Douglas JM, et al. Trends in clinic visits and diagnosed Chlamydia trachomatis and Neisseria gonorrhoeae infections after the introduction of a copayment in a sexually transmitted infection clinic. Sex Transm Dis 2005;32:243-6.

23. Levine DK, Woodriff AJ, Mocello AR, et al. inSPOT: the first online STD partne notification system using electronic postcards. PLoS Med 2008;5:1428-31.

24. Bourne C, Knight V, Guy R, et al. SMS reminder intervention doubles STI/HIV re-testing rates among men who have sex with men. Sex Transm Infect 2011;87:229-31 